\title{
Bismuth-Doped Fiber Integrated Ring Laser Mode-Locked with a Nanotube-Based Saturable Absorber
}

\author{
E. J. R. Kelleher ${ }^{1 *}$, J. C. Travers ${ }^{1}$, Z. Sun ${ }^{2}$, A. C. Ferrari ${ }^{2}$, K. M. Golant ${ }^{3}$, \\ S. V. Popov ${ }^{1}$ and J. R. Taylor ${ }^{1}$ \\ ${ }^{I}$ Femtosecond Optics Group, Physics Department, Imperial College London, \\ Prince Consort Road, London, SW7 2AZ, UK \\ ${ }^{2}$ Department of Engineering, University of Cambridge, Cambridge, $C B 3$ OFA, UK \\ ${ }^{3}$ Kotel'nikov Institute of Radio Engineering and Electronics, Mokhovaya 11-7, Moscow, 125009, Russia \\ *edmund.kelleher08@imperial.ac.uk
}

\begin{abstract}
We demonstrate passive mode-locking of a bismuth-doped fiber laser using a singlewall nanotube-based saturable absorber. Stable operation in the all-normal dispersion and average soliton regime is obtained, with an all-fiber integrated format.

(C)2010 Optical Society of America

OCIS codes: (140.4050) Mode-locked lasers; (140.3510) Lasers, fiber; (140.3560) Lasers, ring
\end{abstract}

\section{Introduction}

There is significant interest in the use of carbon nanotubes as saturable absorbers for mode-locking fiber lasers because of advantageous properties such as wideband operation from a single device [1], highly-engineerable spectral coverage [2], and short recovery times supporting short-pulse generation. To-date, all fiber-based modelocked systems employing single-wall nanotubes (SWNTs) have used well-established rare-earth doped active fibers such as $\mathrm{Yb}, \mathrm{Er}$, and Tm operating around $1 \mu \mathrm{m}, 1.5 \mu \mathrm{m}$ and $1.9 \mu \mathrm{m}$ [2]. The need to extend the range of available wavelengths has driven research into the development of new fiber gain media [3].

Near infrared luminescence from a silica glass fiber doped with bismuth ions was first reported by Dvoyrin et al. in 2005 [3], prompting the first demonstration of a CW laser utilising the new gain medium [4]. Although microscopic origin of the IR luminescence and lasing in bismuth-doped (Bi-doped) silica remains unclear, a broad emission band in the region $1.1 \mu \mathrm{m}-1.25 \mu \mathrm{m}$ makes Bi-doped fiber an attractive medium for the generation of short pulses [5]. Here we demonstrate passive mode-locking of a Bi-doped fiber laser using a nanotube-based saturable absorber, with dispersion compensation provided by a strongly chirped FBG allowing an all-fiber format. To the best of our knowledge, this is the first application of SWNTs as a saturable absorber element for passive mode-locking of a Bi-doped fiber laser.

\section{Experimental setup}

The all-fiber geometry is shown schematically in Fig. 1. We tested two cavity configurations: cavity A, with a net normal dispersion due to the Bi-doped fiber, and cavity $\mathrm{B}$, compensated to achieve net anomalous dispersion. The Bi-doped fiber was fabricated with using SPCVD [6]. Features of this plasma chemical technology applied to the fabrication of Bi-doped silica fiber preforms, can be found in [7]. The relatively low pump absorption and gain coefficient of the currently available Bi-doped fiber sample meant that a $30 \mathrm{~m}$ length was required to obtain lasing. In addition, we observed that the gain had strong temperature dependence: it was enhanced when the bismuth fiber was cooled to $77 \mathrm{~K}$, which we did for all of the results presented here. The active fiber was core-pumped through a custom wavelength division multiplexer (WDM) with a commercial $10 \mathrm{~W}$ Yb-fiber laser at $1.065 \mu \mathrm{m}$. The modelocking threshold was typically $250 \mathrm{~mW}$ pump power.
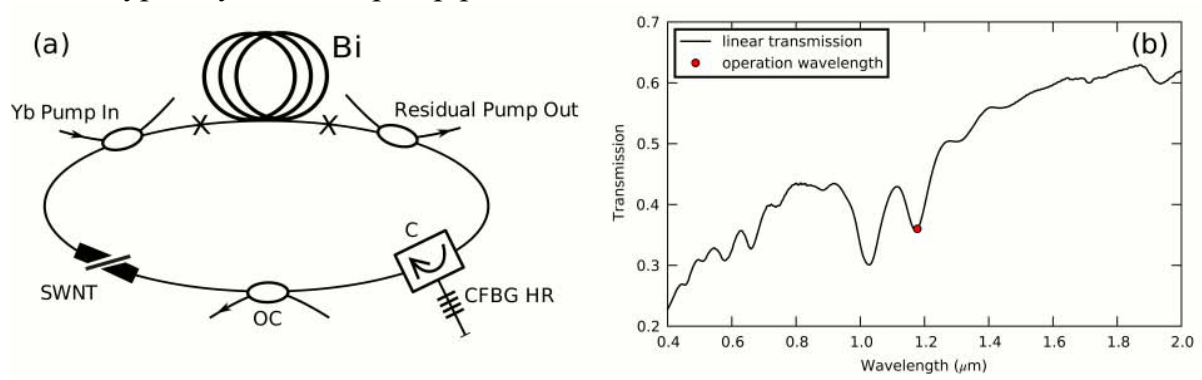

Figure 1. (a) Bi-doped fiber laser setup. Bi, bismuth-doped active fiber; C, circulator; CFBG HR, chirped fiber Bragg grating highreflector; OC, output-coupler; SWNT, nanotube-based saturable absorber. (b) linear transmission spectrum of the SWNT-based saturable absorber composite film, with a strong absorption peak engineered to coincide with the operating wavelength (indentified with a red circle). 


\section{CTuII4.pdf}

The residual pump light was coupled out of the laser using a second WDM. In cavity configuration A, we used a fiber pigtailed isolator to maintain unidirectional operation, and a $20 \%$ output coupler. In cavity B, dispersion compensation was provided by a CFBG incorporated into the cavity using a fiber pigtailed optical circulator, which also imposed unidirectional propagation. Due to the higher loss of the circulator the output coupler was reduced to $5 \%$ to minimize the cavity loss. Finally, in both configurations, the SWNT saturable absorber film was integrated using two APC fiber connectors, suffering typically $3.5 \mathrm{~dB}$ insertion loss with $2 \mathrm{~dB}$ attributed to the linear transmission of the composite (see Fig. 2). The SWNT saturable absorber was fabricated using a technique discussed in [2] and references therein.

\section{Results and discussion}

Self-starting mode-locked operation was obtained in both cavity configurations, with a $5 \mathrm{MHz}$ repetition rate. In configuration $\mathrm{A}$, the net normal dispersion supported 600 ps dissipative solitons, carrying a significant chirp [8]. It is worth noting, because of recent notable interest in this giant-chirp regime for access to high pulse energiesthat the average output power was $10 \mathrm{~mW}$, with pulse energies of $2 \mathrm{~nJ}$. Such high-powers were only possible because the total round-trip loss of the cavity was lower, without the inclusion of the circulator, and therefore the higher output coupling ratio of $20 \%$ could be supported.

With the inclusion of the circulator and CFBG (cavity B), we obtained average soliton operation. Figure 3(a) shows the output spectrum, with prominent solitonic sidebands and a full width at half maximum (FWHM) bandwidth of $0.35 \mathrm{~nm}$. The additional peaks on the long-wavelength edge of the spectrum are caused by high-order dispersion due to the CFBG. Figure 3(b) is the corresponding intensity autocorrelation trace, fitted with the autocorrelation shape expected from a sech ${ }^{2}$ pulse. The pulse duration was $4.7 \mathrm{ps}$, giving a time-bandwidth product of 0.36 , near transform limited for a $\operatorname{sech}^{2}$ pulse shape. Although reliable $\mathrm{CW}$ mode-locking was achieved for a fixed pump power, because of the low gain coefficient of the active fiber and high modulation depth of the saturable absorber the system would become unstable with elements of Q-switching for increased pump power.
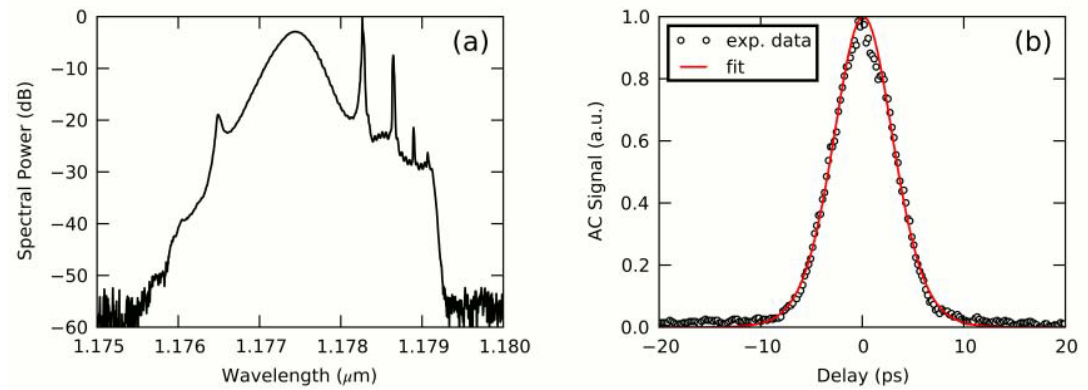

Figure 3. (a) the optical spectrum and (b) corresponding intensity autocorrelation, with fit (red).

In conclusion, we demonstrated the first Bi-doped fiber laser mode-locked with a nanotube-based saturable absorber. Stable, self-starting mode-locking was achieved in a normally and anomalously dispersive cavity with pulse durations of 600 and $4.7 \mathrm{ps,} \mathrm{respectively} \mathrm{at} \mathrm{a} \mathrm{repetition} \mathrm{rate} \mathrm{of} 5 \mathrm{MHz}$, dictated by the long length of gain fiber required for sufficient pump absorption. Further improvement of the SPCVD process would make it possible to use shorter fiber lengths. Wavelength diversity provided by the broad gain profile of bismuth, and alternative schemes to compensate the cavity dispersion will be discussed.

\section{References}

[1] F. Wang, A. G. Rozhin, V. Scardaci, Z. Sun, F. Hennrich, I. H. White, W. I. Milne and A. C. Ferrari, "Wideband-tuneable, nanotube modelocked, fibre laser", Nat. Nano. 3, 738 (2008).

[2] T. Hasan, Z. Sun, F. Wang, F. Bonaccorso, P. H. Tan, A. G. Rozhin and A. C. Ferrari, "Nanotube-Polymer Composites for Ultrafast Photonics", Adv. Materials 21, 3874 (2009).

[3] V. V. Dvoyrin, V. M. Mashinsky, E. M. Dianov, A. A. Umnikov, M. V. Yashkov and A. N. Guryanov, "Absorption, fluorescence and optical amplification in MCVD bismuth-doped silica glass optical fibres" in Optical Communication, ECOC 2005. $31^{\text {st }}$ European Conference on, 4949 (2005).

[4] E. M. Dianov, V. V. Dvoyrin, V. M. Mashinsky, A. A. Umnikov, M. V. Yashkov and A. N. Gur'yanov, "CW bismuth fiber laser”, Quan. Elec. 35, 1083 (2005).

[5] S. Kivistö, R. Gumenyuk, J. Puustinen, M. Guina, E. M. Dianov and O. G. Okhotnikov, "Mode-Locked Bi-Doped All-Fiber Laser With Chirped Fiber Bragg Grating", IEEE Phot. Tech. Lett. 21, 599 (2009).

[6] D. Pavy, M. Moisan, S. Saada, P. Chollet, P. Leprince, and J. Marrec, "Fabrication of optical fiber preforms by a new surfaceplasma CVD process," in Proceedings of 12th European Conference on Optical Communications, pp. 19-22. (Barcelona, 1986),

[7] I. A. Bufetov, K. M. Golant, S. V. Firstov, A. V. Kholodkov, A. V. Shubin, and E. M. Dianov, "Bismuth activated alumosilicate optical fibers fabricated by surface-plasma chemical vapour deposition technology", Appl. Optics, 47, 4940 (2008).

[8] E. J. R. Kelleher, J. C. Travers, E. P. Ippen, Z. Sun, A. C. Ferrari, S. V. Popov and J. R. Taylor, "Generation and direct measurement of giant chirp in a passively mode-locked laser", Opt. Lett. 34, 3526 (2009). 